\title{
A fuzzy credibility model to estimate the operational value at risk using internal and external data of risk events
}

\author{
Alejandro Peña ${ }^{\mathrm{a}, *}$, Isis Bonet ${ }^{\mathrm{a}}$, Christian Lochmuller ${ }^{\mathrm{a}}$, Héctor Alejandro Patiño ${ }^{\mathrm{a}}$, Francisco \\ Chiclana $^{\mathrm{b}}$, Mario Góngorab \\ ${ }^{a}$ Computational Modelling and Simulation (GISMOC and GPC) Research Group, EIA University, Envigado, \\ Colombia \\ ${ }^{b}$ Centre for Computational Intelligence (CCI), De Montfort University, Leicester, England
}

\begin{abstract}
Operational Risk (OpR) refers to the possibility of suffering losses resulting from inadequate or failure of processes and/or technology, inadequate behaviour of people or external events. OpR was one of the main risks that led to the 2008 global financial crisis. Limitations of the analytical models that are applied in estimating this risk surface when qualitative information, frequently associated with OpR events, is used. To determine the magnitude of OpR in financial organisations, qualitative datainnd also historical data from risk events can be used. Current research trends that focus on the development of analytical models, by using different databases, to estimate the Operational Value at Risk (OpVaR) still lack models based on qualitative information, risk management profiles and the ability to integrate different databases of OpR events. In this paper we present a fuzzy model to estimate the OpVaR of an organisation by working with two different databases that contain internal available data and external or observed data. The proposed model considers: (1) the intrinsic properties of the data as fuzzy sets related to the linguistic variables of the observed data (external) and the data from available databases (internal), and (2) a series of management profiles to mitigate the effect that external data usually causes in estimating the $\mathrm{OpVaR}$ of an organisation. The results obtained with the proposed model allow an organisation to estimate and determine the behaviour of the OpVaR over time by using different risk profiles. The integration of qualitative information, different risk profiles (ranging from weak to strong risk management), and internal and external databases contributes to the advancement of estimating the OpVaR in risk management.
\end{abstract}

Keywords: Operational Value at Risk (OpVaR), Risk profile, Basel II, Loss Distribution Approach (LDA), Fuzzy credibility model, internal and external data

\section{Introduction}

Operational Risk (OpR), which was one of the risks that led to the world financial crisis in 2008, is defined by the Basel II committee as "the risk of loss resulting from inadequate or failed internal processes, people and systems or from external events" [1, page 3]. This same agreement mentions that "any operational risk measurement system must [...] include the use of internal data, relevant external data, scenario analysis and factors reflecting the business environment and internal control systems." [2, pages 45-46]. The limitations of the analytical models that are applied to estimate the Operational Value at Risk (OpVaR) become evident

\footnotetext{
*Corresponding author

Email addresses: pfjapena@gmail.com (Alejandro Peña), ibonetc@gmail.com (Isis Bonet), christian.lochmuller@eia.edu.co (Christian Lochmuller), alepaper@gmail.com (Héctor Alejandro Patiño), chiclana@dmu.ac.uk (Francisco Chiclana), mgongora@dmu.ac.uk (Mario Góngora)
} 
when using a significant amount of qualitative information, such as the information used to describe failures in a business process of an organisation, including financial institutions [3]. To achieve the required confidence level in estimating the OpVaR, many organisations have to use external databases and scenarios of OpR events. However, this data, in many cases, does not represent the reality of the modelling organisation [4]. Thus, there are two main question to address that refer, on the one hand, to the credibility of the external data used in estimating the OpVaR in comparison to the historical, internal OpR event data of an organisation, and, on the other hand, to the evolution of this risk over time, when using different risk management profiles, ranking from weak to strong risk management, that might impact the expected losses in the future [5].

Four development research trends can be identified when revising the literature on this knowledge area:

The first research trend is related to the estimation of the OpVaR by using different models and techniques based on the definitions of the Basel II agreement [6, 7]. After the publication of the Basel II agreement, the most common models used to estimate the OpVaR are the Standardized Indicator (SI), the Basic Indicator (BI) and the Advanced Measurement Approach (AMA) [8]. In particular, AMA based models are quantitative OpR models that use internal and external data [9], and they are widely used. However, their role in the recent world financial crisis has been picked up by some scholars [10]. All these models have to consider the following key elements, which are subject to management decisions, related to OpR: causes, events, controls and impact in the estimation of OpVaR; in order to mitigate or remove this type of risk to some extent from an organisation by using, for example, management matrices [11]. Over time, this research trend has established new approaches for the estimation of the OpVaR in financial institutions and in using risk management policies that consider the conditions of the international financial markets [12]. Accordingly, mathematical and statistical models can be highlighted that implement different methods and techniques [13] that use internal and external data, scenarios and risk management matrices in AMA models, based on the definitions of the Basel II Agreement.

The second research trend estimates the OpVaR by using Bayesian models. Among them, a model stands out that uses the Bayesian approach to integrate three different sources of information [14] to estimate the OpVaR, identifying the causes and the relationship between risk events [15]. Also, a new methodology has been proposed to frame risk selfassessment data into suitable prior distributions, updated with observed loss data. This produces posterior distributions from which the OpVaR can be determined accurately [16]. Furthermore, this Bayesian approach has also been used to identify and quantify the OpR associated with financial transactions using electronic devices [17]. This research line aims at understanding the causes and the relationship between risk events in order to integrate into one single model internal and external data and the application of credibility theory that is based on Bayesian concepts [18].

The third research trend integrates internal and external databases with risk events by using different credibility theory techniques. In [19], different techniques are used to create scenarios and external risk events that reflect the market or risk occurrences that happened in other financial institutions. These quantitative models combine internal and external data to overcome the limitation of modelling only internal risk event data only, which refers to the volume of data available, because extreme OpR events rarely occur. Thus, internal data is mixed with external data so that tail events can be modelled [20, 21]. To overcome the limitation of not possessing sufficient internal data, other authors [22] 
proposed the use of scenarios to model extreme events, which reside in the tail of the loss distributions, so that the body and the tail of the loss distribution can be modelled separately. Moreover, models have been proposed that integrate databases by using a novel approach of multidimensional credibility, which is based on Bühlmann credibility theory [23-25]. This research line applies the definitions of Basel II, which requires that AMA models for estimating the OpVaR of an organisation should include internal, external and scenario data, but also data related to the business environment and internal control factors (BECF).

The fourth research trend focuses on the developing of fuzzy logic based models to estimate the OpVaR. An evaluation of the OpR by means of a fuzzy inference model to manage endogenous and exogenous risk factors was presented in [26]. In that context, the fuzzy C-means and fuzzy swarm for fuzzy clustering have been applied to identify the relevant data in the databases [27] and to show how to organise risk event type variables into macro classes based on fuzzy variables to improve OpR management [28]. Additionally, models can be highlighted that use fuzzy logic to integrate different databases to estimate the OpVaR [3] and a checklist-based fuzzy weighted severity approach for calculating OpR exposure with regard to foreign exchange trades under Basel II [29]. These works show that this line of research focuses on the integration of internal and external data and aiming at improving the estimation of the OpVaR by incorporating highly qualitative information [30]. However, there is still a lack of proposals that estimate the OpVaR by using both internal and external data of risk events, considering the intrinsic quantitative and qualitative properties of the data and risk management matrices. This is the focus of the present article and, as further elaborated below, a novel fuzzy credibility model to estimate the $\mathrm{OpVaR}$ is developed by integrating two databases representing the internal available data $(\mathrm{AD})$ and external or observed $(\mathrm{OD})$ data of $\mathrm{OpR}$ events in a financial organisation.

Fuzzy logic and methods can also be found in other risk management areas, with the field of emergency decision making (EDM) related to natural disasters being an example [35]. In this case, a natural disaster represents an OpR event that may cause damage to physical assets of an organisation. Disaster management requires risk management, primarily related to extreme events, and both strive to improve decision making. In both knowledge areas, decision making for OpR management and emergency decision making, fuzzy set theory has been applied because decision information is often vague or incomplete [31-34], which is most notably true in emergency situations [36]. With regard to the integration of data and information from different sources, in [36] the authors also highlight, in the context of EDM, that at present "weight determination methods are almost confined to the static weights and few of them have been introduced into dynamic intuitionistic fuzzy decision making", which reinforces the necessity of taking into account the dynamic changes of the influencing factors in the estimation of the OpVaR.

This paper presents, with regard to the above fourth trend of research, a novel fuzzy credibility model to estimate the OpVaR through the integration of two databases that represent both the internal available data (AD) and external or observed (OD) data of OpR events in a financial organisation. The proposed model is composed of two submodels, with the first one allowing the estimation of the credibility of each database. This is done by using the overlap between fuzzy sets to represent the quantitative and qualitative intrinsic properties for the AD and OD as linguistic variables (ADLV - ODLV). To estimate the credibility, it is necessary to establish a series of definitions, identifying this way the presence of distributions with long tails, according to the form and shape of the fuzzy sets. The second submodel allows to estimate the OpVaR by using the credibility and different risk management profiles for the OD, so that 
an $m$ fuzzy risk management model is configured. Additionally, this model allows to assess the evolution of the loss distribution (LD) by using a sequence of Fuzzy Risk Management Matrices (FRMMs). The proposed model achieves both lower OpVaR values and a narrower LD than the analytical models based on Bühlmann credibility theory, as shown in Section 4. This is mainly due to the control that the FRMMs exert on the risk events that conform the OD database. Thus, main contribution of this paper is the development of a model able to integrate qualitative information, different risk profiles (ranging from weak to strong risk management) and databases in estimating the OpVaR in risk management.

The rest of the article is organised as follows. Section 2 provides the background theory related to the estimation of the $\mathrm{OpVaR}$ (Section 2.1) and to the analytical credibility estimator based on Bühlmann credibility theory (Section 2.2). Section 3 presents the structure of the proposed $\mathrm{m}$ _fuzzy model and the experimental design to analyse and validate its behaviour. Section 4 analyses a series of results regarding the behaviour of the model with regard to the estimation of the $\mathrm{OpVaR}$ by using different risk profiles. In Section 5 the most important conclusions are presented and recommendations are made with respect to future work in this knowledge area.

\section{Theory}

\subsection{OpR Modelling}

In general, there are three different methods available for financial organisations to estimate the OpR [37]: (1) the Basic Indicator Approach (BIA) allows to estimate the OpR applying a fixed rate of $15 \%$ to the gross annual income during the three previous years; (2) the Standardized Approach (SA) is less general and allows to estimate the OpR by using a percentage, referred to as beta factor, of the annual gross income for each of the eight business lines. This way, the beta factor weights the riskiness of a business line; and (3) the Advanced Measurement Approach (AMA), which includes analytical models that use quantitative and qualitative criteria to estimate the OpR and the regulatory capital for an organisation. In essence, the first two methods determine the regulatory capital by using a fixed percentage of the gross annual income, with the main difference between them being that that the second one requires the sum of the capital needs per line of business [38]. These two models determine the operational risk simply by relating it to the annual gross income, which represents a very general way to determine the level of $\mathrm{OpR}$ and in consequence the required regulatory capital of a financial institution, whereas the AMA models use historical observed data. The OpVaR in the context of AMA models is defined according to the definitions of the Basel Committee on Bank Supervision (BCBS) as the maximum loss that can be expected given a certain confidence level $(\alpha)$ and within a certain period of time (typically one year) for OpR. The OpVaR value is obtained from the loss distribution (LD), which reflects the probability of occurrence of a risk event in a business line in an organisation with a confidence level of 99.9\% [37]. The two variables parameters that allow the identification of the losses are severity, which is the amount of loss registered in the analysed period of time, and frequency, which refers to how often a risk event occurs in this same period of time. The LD distribution is obtained through the convolution of these random variables (frequency and severity) by using different statistical sampling methods [13]. The LD distribution has three different representative values as represented in Figure 1 [3]: (i) expected losses (EL), which represent the set of losses below the mean; (ii) the Op VaR as described above, which identifies the severe losses; and (iii) unexpected losses (UL), which are located between the mean and the OpVaR.

\subsection{The Bühlmann Credibility Estimator}

Due to the limited quantity of internal loss data generated through OpR events, organisations need to use external data to achieve the required degree of confidence for the estimation 


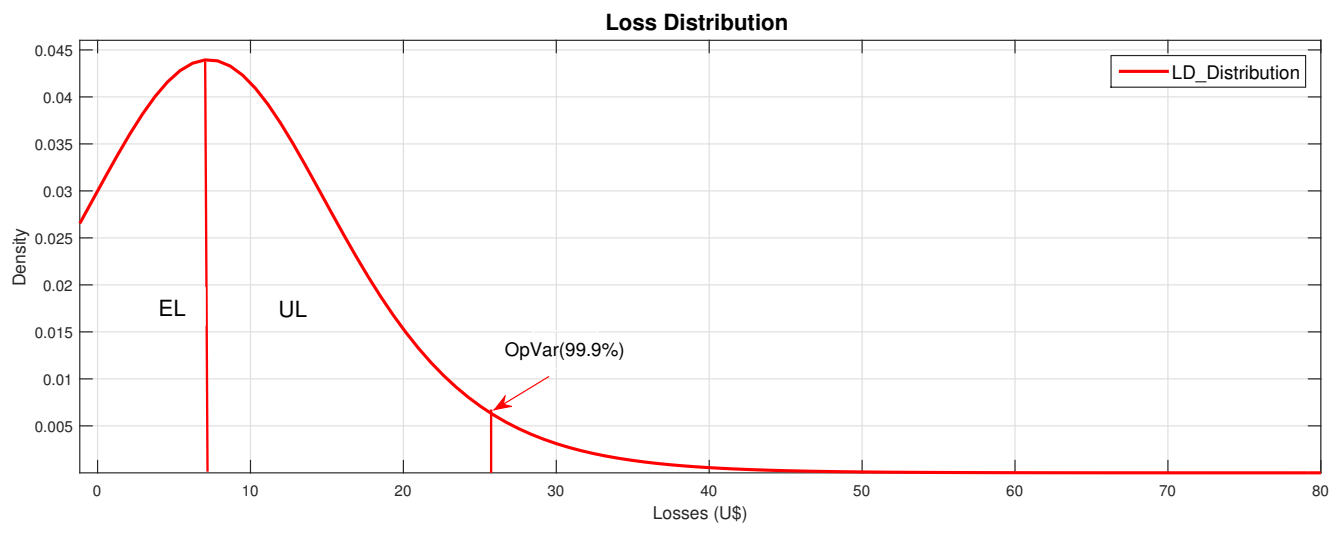

Figure 1: General structure of the loss distribution (LD)

of the OpVaR (99.9\% is the value defined by the Basel II Accord [37]). In order to use internal and external data in an OpR model these data sources are "mixed" according to their respective level of credibility. Credibility is defined as the degree of accuracy in forecasting statistically future events, based on a set of reported past or historical risk events, where the weight assigned to the observed internal data is defined relative to the external data through analytical methods or by judgement. The weight or credibility assigned to the observed data increases with the number of records and decreases with higher levels of variability in the data [39].

Three statistical models to estimate the credibility from observed historical risk events can be highlighted: least square credibility, empirical bayesian credibility and the Bühlmann credibility. Models based on least square credibility are most commonly used. These models attempt to produce linear estimates that minimise the square of differences between the estimated value of risk and the quantity being estimated. However, the Bayesian and Bühlmann credibility models have been used most in recent years, thanks to their capacity to work with the intrinsic properties of the data when the underlying distributions are unknown [17].

Given $X_{1}, X_{2}, \ldots, X_{n}$ as the historical loss amounts that represent failures occurred in business processes, and assuming these to be independent and identically distributed, where the distribution of the risk characteristics in the population of the data being represented by $\pi_{\theta}(\theta)$, the Bühlmann credibility estimate for the $O p V a R_{k+1}$ is $[3,40]$ :

$$
O p V a R_{k+1}=Z \cdot \bar{X}+(1-Z) \cdot \mu,
$$

where $\bar{X}$ is the mean of the historical loss amounts; $\mu$ is the expected value of the hypothetical mean, i.e. the unconditional mean $E[X]$, using a risk parameter of $\theta$ or a particular type of risk event.

$$
\mu=E[X]=E[E[X \mid \theta]]
$$

$Z$ is the credibility factor assigned to the observed or available data (AD)

$$
Z=\frac{N}{N+k}
$$

with $N$ representing the number of observed historical risk events. The Bühlmann-Straub credibility factor $(k)$ is defined:

$$
k=\frac{E P V}{V H M},
$$

where $E P V$ is the expected process variance,

$$
E P V=E[\operatorname{Var}[X \mid \theta]],
$$


and $V H M$ is the variance of the hypothetical means,

$$
V H M=\operatorname{Var}[E[X \mid \theta]] .
$$

The total variance of this random processes is:

$$
\operatorname{Var}[X]=E[\operatorname{Var}[X \mid \theta]]+\operatorname{Var}[E[X \mid \theta]] .
$$

The Bühlmann credibility for estimating the OpVaR is a linear model of available past data that takes the following form:

$$
\begin{gathered}
O p V a R_{k+1}=Z \cdot \bar{X}+(1-Z) \cdot \mu=w_{o}+\sum_{k=1}^{N D} w_{k} \cdot x_{k} \\
w_{o}=(1-Z) \cdot \mu \\
w_{k}=\frac{Z}{n} ; \quad k=1,2,3, \ldots, N D
\end{gathered}
$$

with $N D$ indicating the number of available data (AD). This linear model is the best linear estimator of the Bayesian predictive mean, $E\left[x_{k+1} \mid x_{1}, x_{2}, \ldots, x_{n d}\right]$, and the hypothetical mean, $E\left[x_{k+1} \mid \theta\right]$, that minimises the square error loss. The $w_{i}$ coefficients are obtained by minimising the loss functions over all $\mathrm{AD}$ events for a parameter $\theta[41]$ :

$$
\begin{gathered}
L_{1}=E\left(\left[E\left[x_{k+1} \mid \theta\right]-w_{0}-\sum_{k=1}^{N D} w_{k} \cdot x_{k}\right]^{2}\right) \\
L_{2}=E\left(\left[E\left[x_{k+1} \mid x_{1}, x_{2}, \ldots, x_{k}\right]-w_{o}-\sum_{k=1}^{N D} w_{k} \cdot x_{k}\right]^{2}\right)
\end{gathered}
$$

These loss functions can be obtained by using fuzzy stochastic neuronal network models [42], based on an evolutionary polynomial model [39] with estimation distribution algorithms [43].

\section{Methodology}

One of the main issues to address when estimating the OpVaR in a financial organisation is related to the characteristics of the $\mathrm{AD}$ in the database of risk events (endogenous database). As already mentioned, the OpVaR should be estimated for a confidence level of $99.9 \%$. However, the low frequency with which an OpR event occurs, may lead to databases that do not have sufficient data to achieve such confidence level. To overcome this problem, organisations use external or exogenous databases (OD) that come from other organisations or governmental financial institutions, which in many cases cannot represent the reality of the market or of the financial organisation itself. For this reason, in this paper we propose a fuzzy credibility model to estimate the $O p V a R$ of an organisation by using the qualitative and quantitative intrinsic properties for the OD and AD as linguistic variables. The proposed fuzzy model is analysed and validated by using different risk profiles, which show a priori the evolution of the OpVaR in a financial organisation over time. 


\subsection{Experimental Design}

To design the fuzzy credibility model two databases were used. A first database with available data (AD) that is composed by a 700 daily records of risk events in total related to failures of cash machines (business line retail banking) and that were recorded in a financial organisation during 2009 and 2010. A second database of external/observed data (OD), which consists of 350 records of daily risk events that are related to the same risk and that were recorded during the year 2011. At an initial stage, an analysis was carried out with respect to the structure and shapes of the fuzzy sets that represent the OD and AD databases as linguistic variables (ODLV, ADLV), as well as the credibility associated with the overlaps among fuzzy sets. To analyse the behaviour of the model in estimating both the credibility and the OpVaR, three case studies were carried out in a first stage. These are related to the structure of fuzzy sets that represent both the ODLV and ADLV. For these variables, 16 ODs were constructed by using different impact factors that show the compression and expansion of losses in an OD database. At a second stage, the analysis and validation of the proposed fuzzy model was performed by using different risk management matrices or risk profiles for the OD, configuring an $m$ fuzzy credibility model that allows us to assess the evolution of the OpVaR in a financial organisation over time [44].

\subsection{Characterization of the Fuzzy Sets}

One of the main issues in credibility theory when estimating the OpVaR is related to the intrinsic statistical properties of the data. In this sense, the intrinsic statistical properties of a database represented as a linguistic variables require the following definitions.

Definition 1. Let $X_{1}, X_{2}, \ldots, X_{N D}$ be records of loss amounts that have been stored in a database of risk events, $q_{0}, q_{1}, q_{2}, q_{3}, q_{4}$ the quantiles of the data, and $X C_{0}, X C_{1}, X C_{2}, X C_{3}, X C_{4}$ the clusters of the data. The intrinsic characteristics of the data are given by the linear regression:

$$
q_{i}=m \cdot X C_{i}+b
$$

where $b$ represents the intercept with the y-axis; and $m$ represents the slopes of the line.

Accordingly, the experimental distribution has the following intrinsic statistical characteristics in terms of the slope:

$m=1:$ The clusters that represent the fuzzy sets are uniformly distributed (balanced fuzzy sets).

$m>1$ : The clusters that represent the fuzzy sets are located toward the left side of the horizontal axis. This indicates the presence of distributions with long tails with unbalanced fuzzy sets.

$m<1$ : The clusters that represent the fuzzy sets are located toward the right side of the horizontal axis. This indicates the presence of negative asymmetry with unbalanced fuzzy sets.

The Bühlmann credibility factor allows to estimate the relation between the intrinsic properties of the observed data and the intrinsic properties of the available data.

Definition 2. The credibility factor is defined as follows:

$$
J=\frac{N}{N+k}
$$


where $N$ indicates the number of observed risk events; $k$ is the Bühlmann credibility factor

$$
k=\frac{E P V}{V H M}
$$

with $E P V$ being the expected value of process variance $(E P V=E[\operatorname{Var}[X[\theta]]])$; and $\operatorname{VHM}$ the variance of the hypothetical means $(\operatorname{VHM}=\operatorname{Var}[E[\theta]])$.

The impact factor allows the expansion or contraction of the fuzzy sets associated with an ODLV.

Definition 3. The impact factor $(I F)$ is defined as follows:

$$
\begin{gathered}
X C_{O D, j, i, k+1}=I F \cdot X C_{O D, j, i, k} \\
\sigma_{O D, j, k+1}=I F \cdot \sigma_{O D, j, k},
\end{gathered}
$$

where $X C_{O D, j, i, k}$ represents the component $i$ of the fuzzy set $j$ in instant $k ; \sigma_{O D, j, k}$ represents the standard deviation of the data in the cluster $j$. IF values in the interval $(0,1)$ allow the contraction of the fuzzy sets, while values in the interval $(1, \infty)$ allows the expansion of the fuzzy sets.

\subsection{The Fuzzy Risk Management Matrix (FRMM)}

The FRMM is a data structure established according to the BCBS definitions to manage OpR. Each row of this matrix is defined by the labels that describe the OD database in terms of a linguistic variable (ODLV), while each column is defined by the labels that describe the AD database, also in terms of a linguistic variable (ADLV). The linguistic variables are obtained by using the fuzzy c-means clustering method [45], where each element of the matrix is defined by a value that shows both the mixed impact of two fuzzy sets on the OpVaR and a "value of management" to reduce this impact. The FRMM is defined as [46]:

$$
\mathrm{FRMM}=\left[\begin{array}{cccc}
\left(m i_{1,1}, m g_{1,1}\right) & \left(m i_{1,2}, m g_{1,2}\right) & \cdots & \left(m i_{1, n c}, m g_{1, n c}\right) \\
\left(m i_{2,1}, m g_{2,1}\right) & \left(m i_{2,2}, m g_{2,2}\right) & \cdots & \left(m i_{2, n c}, m g_{2, n c}\right) \\
\vdots & \vdots & \vdots & \vdots \\
\left(m i_{n c, 1}, m g_{n c, 1}\right) & \left(m i_{n c, 2}, m g_{n c, 2}\right) & \cdots & \left(m i_{n c, n c}, m g_{n c, n c}\right)
\end{array}\right]
$$

where $n c$ is the number of labels that describe the ODLV and ADLV; $m i_{l, j}$ represents the combined impact on the OpVaR of the $l$ fuzzy ODLV label and the $j$ fuzzy ADLV label; $m g_{l, j}$ represents the level or degree of management to reduce the combined impact aforementioned; $l=1,2,3,4,5$ (Very Low, Low, Medium, High, Very High); and $j=1,2,3,4,5$ (Very Low, Low, Medium, High, Very High).

To evaluate the behaviour of the model we propose three different fuzzy risk management matrices, which can be applied for different scenarios that define the $I F$. Figure 2 shows three risk profiles of management with regard to OD events, where (a) represents a weak management with minor values for the impact, (b) shows a balanced management, where the level of impact is balanced with the levels of management, and (c) showing higher levels of management than levels of impact. The sequence E1 - E2 - E3 represents a development path for an organisation to achieve a strong risk management [47]. 


\begin{tabular}{|c|c|c|c|c|c|c|}
\hline & \multicolumn{5}{|c|}{ AD Fuzzy Sets } \\
\hline & & Very Low & Low & Medium & High & Very High \\
\hline \multirow{5}{*}{ 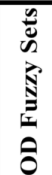 } & Very High & 0.5 & 0.5 & 1 & 1 & 1 \\
\hline & High & 0.5 & 1 & 1.5 & 1.5 & 1.5 \\
\hline & Medium & 0.5 & 1 & 1.5 & 1.5 & 1.5 \\
\hline & Low & 1 & 1 & 1.5 & 2 & 2 \\
\hline & Very Low & 1 & 1 & 1.5 & 2 & 2.5 \\
\hline
\end{tabular}

(a)

\begin{tabular}{|c|c|c|c|c|c|c|}
\hline & \multicolumn{5}{|c|}{ AD Fuzzy Sets - Weak Management } \\
\hline & & Very Low & Low & Medium & High & Very High \\
\hline \multirow{5}{*}{ 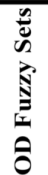 } & Very High & 1 & 1 & 1 & 1 & 1 \\
\hline & High & 1 & 2 & 2 & 2 & 2 \\
\hline & Medium & 1 & 2 & 3 & 3 & 3 \\
\hline & Low & 1 & 2 & 3 & 4 & 4 \\
\hline & Very Low & 1 & 2 & 3 & 4 & 5 \\
\hline
\end{tabular}

(b)

\begin{tabular}{|c|c|c|c|c|c|c|}
\hline & \multicolumn{5}{|c|}{ AD Fuzzy Sets - Strong Management } \\
\hline & & Very Low & Low & Medium & High & Very High \\
\hline \multirow{5}{*}{ 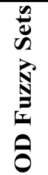 } & Very High & 3 & 3 & 3 & 3 & 3 \\
\hline & High & 3 & 4 & 4 & 4 & 4 \\
\hline & Medium & 3 & 4 & 6 & 6 & 6 \\
\hline & Low & 3 & 4 & 6 & 7 & 7 \\
\hline & Very Low & 3 & 4 & 6 & 7 & 9 \\
\hline
\end{tabular}

(c)

\begin{tabular}{|l|l|c|c|c|}
\hline \multicolumn{5}{|c|}{ Impact Levels } \\
\hline 1 & 2 & 3 & 4 & 5 \\
\hline
\end{tabular}

Figure 2: Fuzzy risk management matrices: (a) Weak management (E1); (b) Balanced management (E2); (c) Strong Management (E3).

\subsection{Fuzzy Credibility Model (FCM)}

To estimate OpVaR at a confidence level of $99.9 \%\left(O p V a R_{99.9 \%}\right)$ by using two different databases that characterise the internal (AD) and external (OD) OpR events of a financial organisation, the proposed fuzzy model has two submodels. A first submodel allows us to estimate the credibility by working with two different linguistic variables that represent the AD and OD database. A second submodel allows us to to estimate the $O p V a R_{99.9 \%}$ by using a risk management matrix on the OD data.

\subsubsection{The fuzzy credibility factor $\left(k_{b}\right)$}

The exponential function that represents the degree of membership of an input data to a fuzzy set is defined as follows [48]:

$$
u_{j, k}=\exp \left(-\frac{1}{2} \sum_{i=1}^{n e}\left(\frac{X C_{j, i}-X_{i, k}}{D_{j}}\right)^{2}\right),
$$

where $u_{j, k}$ is the degree of membership of data $k$ with respect to cluster $j ; X C_{j, i}$ represents the component $i$ of the cluster $j ; X_{i, k}$ represents the component $i$ of the data $k$; $D_{j}$ is the standard deviation of data in the cluster $j$; and ne is the number of input variables for data $k$.

The average degree of membership for all $k$ data with respect to the cluster $j$ can be defined as:

$$
u_{j}=\exp \left(-\frac{1}{2 \cdot N D} \sum_{k=1}^{N D}\left(\sum_{i=1}^{n e}\left(\frac{X C_{j, i}-X_{i, k}}{D_{j}}\right)^{2}\right)\right),
$$

where $N D$ is the number of data to be evaluated. 
Considering equation (20), the average degree of membership of $k$ data associated with the cluster $l$ with respect to the centre of the cluster $j$ is defined as:

$$
u_{l, j}=\exp \left(-\frac{1}{2 \cdot N D L} \cdot \frac{1}{D_{j}^{2}} \sum_{k=1}^{N D L}\left(\sum_{i=1}^{n e}\left(X C_{j, i}-X_{l, i, k}\right)^{2}\right)\right),
$$

where $N D L$ is the number of data associated with the cluster $l ; X_{l, i, k}$ represents the component $i$ of the $k$ data associated with the cluster $l$.

From Definition 2, and taking into consideration equation (21), we have:

$$
V H M B_{l, j}=\frac{1}{N D L} \sum_{k=1}^{N D L}\left(\sum_{i=1}^{n e}\left(X C_{j, i}-X_{l, i, k}\right)^{2}\right),
$$

where $V H M B_{j, l}$ is the variance of the data associated with the cluster $l$ of the ODLV with respect to the cluster $j$ of the ADLV.

As the $k$ data belongs to a different linguistic variable that defines the cluster $j$, the EPV can be expressed as:

$$
\begin{gathered}
D_{j}=\frac{\sigma_{j}+\sigma_{l}}{2} \\
E P V B_{l, j}=D_{j}{ }^{2},
\end{gathered}
$$

where $E P V B_{j, l}$ is the average of the standard deviations (unlike to the degree of membership, which uses only one variable, this variable represents the combined standard deviations); $\sigma_{j}$ is the standard deviation of the cluster $j$ associated with the ADLV; and $\sigma_{l}$ is the standard deviation of the cluster $l$ associated with the ODLV.

In compliance with equation (24), the credibility between the fuzzy set $l$ associated with the ODLV with respect to the fuzzy set $j$ associated with the ADLV can be expressed as [40]:

$$
u_{l, j}=\exp \left(-\frac{1}{2} \cdot \frac{V H M B_{l, j}}{E P V B_{l, j}}\right)
$$

where

$$
k_{b, l, j}=\frac{V H M B_{l, j}}{E P V B_{l, j}}
$$

and $k_{b, l, j}$ is the fuzzy credibility between the cluster $l$ for the ODLV and the cluster $j$ for the ADLV.

The fuzzy credibility factor with respect to the cluster $j$ in the AD database can be expressed as:

$$
\begin{gathered}
J_{O D, j}=1-\frac{1}{n c_{O D}} \sum_{l=1}^{n c_{O D}} u_{l, j} \\
J_{A D, j}=\frac{1}{n c_{O D}} \sum_{l=1}^{n c_{O D}} u_{l, j},
\end{gathered}
$$

where $J_{O D, j}$ is the fuzzy credibility factor that represents the average credibility for the ODLV with respect to the cluster $j$ associated with the ADLV.

\subsubsection{The fuzzy credibility structure}

According to the Bühlmann credibility model, the structure of the proposed fuzzy credibility model for estimating the OpVaR by using two different databases ODLV and ADLV is defined as:

$$
O p V a R=\frac{\sum_{j=1}^{n c} J_{O D, j} \cdot X C_{O D, j, i}}{\sum_{j=1}^{n c} J_{O D, j}}+\frac{\sum_{j=1}^{n c} J_{A D, j} \cdot X C_{A D, j, i}}{\sum_{j=1}^{n c} J_{A D, j}}
$$




$$
O p V a R=O p V a R_{O D}+O p V a R_{A D},
$$

where $X C_{O D, j, i}$ is the location of the $j$ cluster using ODLV; $X C_{O D, j, i}$ is the location of the $j$ cluster using ADLV; and

$$
J_{O D j}=\frac{\sum_{l=1}^{n c} \frac{m i_{l, j}}{m g_{l, j}} \cdot u_{l, j}}{\sum_{l=1}^{n c} \frac{m i_{l, j}}{m g_{l, j}}}
$$

The assimilation of the OD data in the AD database is performed through the fuzzy c-means method as follows [45]:

1. Updating the clusters of available data:

$$
X C_{A D, j, i}=\frac{\sum_{k=1}^{N D}\left(u f_{j, i, k}\right)^{m} \cdot x_{O D, i, k}}{\sum_{k=1}^{N D}\left(u f_{j, i, k}\right)^{m}}
$$

where $m$ is the plasticity of the fuzzy sets that constitute a database.

2. The distance between new data $x_{k, i}$ and the fuzzy sets that define the ADLV.

$$
d_{j, k}=\left\|\sqrt{\sum_{i=1}^{n e}\left(x_{k, i}-X C_{A D, j, i}\right)^{2}}\right\|^{2}
$$

3. The update of the fuzzy partition array:

$$
u f_{j, j_{1}, k}=\left(\sum_{j=1}^{N C}\left(\frac{d_{j_{1}, k}}{d_{j, k}}\right)^{\frac{2}{m-1}}\right)^{-1}
$$

The stop criteria allows to identify the assimilation of the OD data in the AD database as: $\left\|U^{k+1}-U^{k}\right\|<5 \times 10^{-p}$, where $p$ indicates the precision of the assimilation.

\subsection{Experimental Validation}

For a general validation of the fuzzy credibility model the following stages apply:

Initial Stage. An analysis was carried out to determine the structure and shape of the ODLV and ADLV, using the associated fuzzy sets. An initial test was also performed to evaluate the credibility in terms of the overlaps between fuzzy sets.

First Stage. An analysis of the behaviour of the model in the estimation of the credibility was carried out by using three different case studies that define the structure and shape of the fuzzy sets that conform the ODLV and ADLV. In the first case study, the ODLV and ADLV were represented using balanced fuzzy sets. In the second case study, the ODLV was represented using balanced fuzzy sets and the ADLV using unbalanced fuzzy sets. In the third case, both ADLV and ODLV were represented using unbalanced fuzzy sets. The unbalanced fuzzy set were obtained using the fuzzy c-means method. For each study 16 ODs were created using an $I F$ which allowed to increase and reduce the losses of the OD database. At this stage the validation of the model was done in terms of the behaviour of the losses using the proposed fuzzy model and the Bühlmann credibility for all ODs and for all structures taken by the linguistic variables. 
Second Stage. An analysis of the behaviour of the proposed fuzzy model was carried out using three different risk management matrices (FRMMs) for the OD data of risk events over time. Furthermore, at this stage 16 ODs were created. The validation and analysis of the proposed model was made based on the evolution of losses by using the Bühlmann model for Mean OpVaR and Variance OpVaR and the proposed fuzzy credibility model applied a set of FRMMs to the 16 OD databases. The set of FRMMs represents the natural evolution of risk in a financial organisation.

\subsubsection{Metrics for credibility}

To analyse and validate the behaviour of the model when estimating the OpVaR the following statistical metrics were used [49, 50]:

1. OpVaR $99.9 \%$ - the OpVaR at a confidence level of $99.9 \%$ with respect to the loss distribution (LD).

2. EL (Expected Losses) - the mean of the LD distribution.

3. UL (Unexpected Losses) - the losses located within the interval [EL,OpVaR $\left.R_{99.9 \%}\right]$.

4. $\sigma$ - the standard deviation of the LD.

5. Negative Loglikelihood - method to estimate the parameters of a statistical model given the observations by finding the parameter values that maximise the likelihood of making the observations given the parameters [51]. Bigger values show a better fit of a probability distribution for the given data.

\subsubsection{Case Study (Canonical Model)}

Aligned with the general structure of the proposed fuzzy credibility model and taking into account the similarity with respect to the losses registered in the OD and AD databases and a weak management matrix (E1), this section presents the estimation of the OpVaR using a canonical model with balanced fuzzy sets for the estimation. Table 1 shows the structure of the balanced fuzzy sets for ODLV and ADLV, while Figure 3 shows the structure and the shapes of the balanced fuzzy sets for ODLV and ADLV, where the slope of the linear regression between quantiles and centroids is $m=1$.

Table 1: Balanced fuzzy sets that represent the Losses in the OD and AD Databases

\begin{tabular}{|c|c|c|c|c|c|}
\hline \multicolumn{7}{|c|}{ Fuzzy Sets - OD Database } \\
\hline & Very Low & Low & Medium & High & Very High \\
\hline $\mathbf{X C}_{\mathbf{j}, \mathbf{i}}$ & 0 & 2.5 & 5 & 7.5 & 10 \\
\hline $\mathbf{D}_{\mathbf{j}}$ & 2.5 & 2.5 & 2.5 & 2.5 & 2.5 \\
\hline \multicolumn{7}{|c|}{ Fuzzy Sets - AD Database } \\
\hline \multicolumn{7}{|c|}{ Very Low } & Low & Medium & High & Very High \\
\hline $\mathbf{X C}_{\mathbf{j}, \mathbf{i}}$ & 0 & 2.5 & 5 & 7.5 & 10 \\
\hline $\mathbf{D}_{\mathbf{j}}$ & 2.5 & 2.5 & 2.5 & 2.5 & 2.5 \\
\hline
\end{tabular}

After the definition of the fuzzy sets for each database, the procedure continues with estimating the overlaps between the fuzzy sets that conform the ODLV and ADLV, which is provided in Table 2). To illustrate the computation process involved, the value corresponding to the fuzzy sets Low for ODLV and Medium for ADLV is explicitly shown:

$$
u_{3,2}=\exp \left(-\frac{1}{2}\left(\frac{5-2.5}{\frac{2.5+2.5}{2}}\right)^{2}\right)=0.607
$$



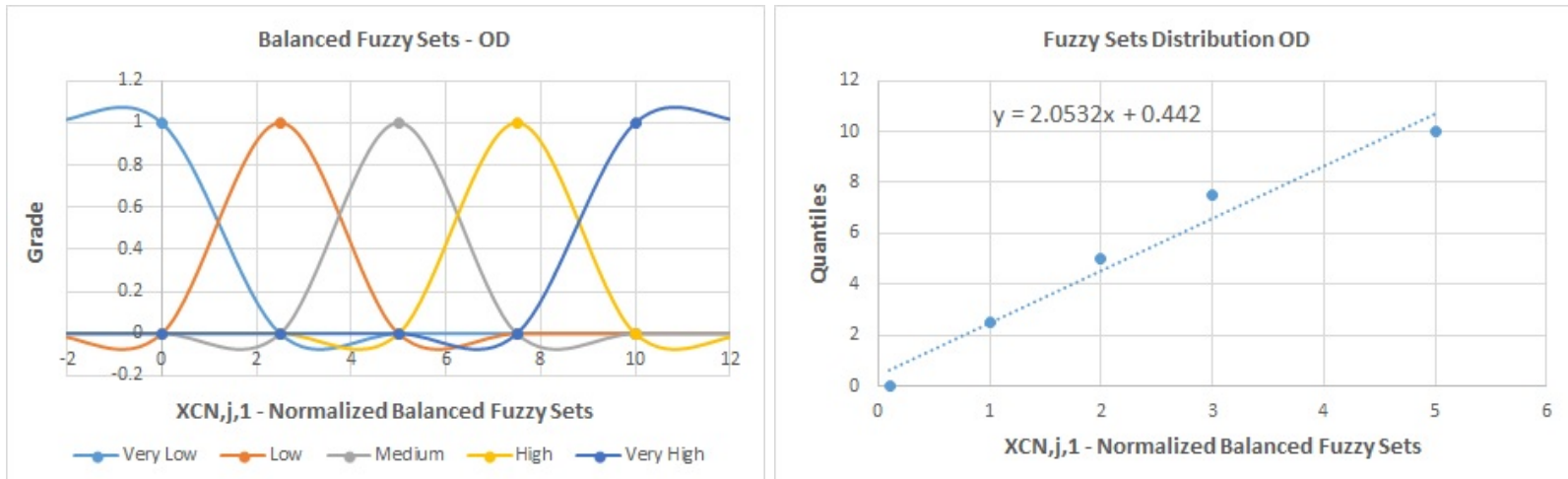

Figure 3: Distribution of the fuzzy linguistic labels for the ODLV and ADLV databases (Balanced Fuzzy Sets)

Table 2: Overlap matrix for the $\mathrm{OD}$ and $\mathrm{AD}$ databases based on balanced fuzzy set

\begin{tabular}{|c|c|c|c|c|}
\hline $\mathbf{u}_{\mathbf{1}, \mathbf{j}}$ & $\mathbf{u}_{\mathbf{2}, \mathbf{j}}$ & $\mathbf{u}_{\mathbf{3}, \mathbf{j}}$ & $\mathbf{u}_{\mathbf{4}, \mathbf{j}}$ & $\mathbf{u}_{\mathbf{5 , j}}$ \\
\hline 1.000 & 0.607 & 0.135 & 0.011 & 0.000 \\
\hline 0.607 & 1.000 & 0.607 & 0.135 & 0.011 \\
\hline 0.135 & 0.607 & 1.000 & 0.607 & 0.135 \\
\hline 0.011 & 0.135 & 0.607 & 1.000 & 0.607 \\
\hline 0.000 & 0.011 & 0.135 & 0.607 & 1.000 \\
\hline
\end{tabular}

The procedure continues with the estimation of the fuzzy credibility for each of the ODLV fuzzy sets as given in Table 3, whose first entry is shown below:

$$
J_{O D, 1}=1-\frac{1}{5}(1.000+0.607+0.135+0.011+0.000)=0.6493
$$

Table 3: Credibility for each fuzzy set that conforms the ODLV and ADLV

\begin{tabular}{|c|c|c|c|c|c|}
\hline & J1 & J2 & J3 & J4 & J5 \\
\hline AD & 0.35066208 & 0.47190112 & 0.49674637 & 0.47190112 & 0.3506620 \\
\hline OD & 0.64933792 & 0.52809888 & 0.50325362 & 0.52809888 & 0.64933792 \\
\hline
\end{tabular}

In accordance with the content of Table 3, the OpVaR estimated by using the structure of the proposed fuzzy model is:

$$
\begin{gathered}
O p V a R_{O D}=\frac{0.649 * 0+0.528 * 2.5+0.503 * 5+0.528 * 7.5+0.649 * 10}{0.649+0.528+0.503+0.528+0.649}=\frac{14.285}{2.857}=5.000 \\
O p V a R_{A D}=\frac{0.351 * 0+0.472 * 2.5+0.497 * 5+0.472 * 7.5+0.351 * 10}{0.351+0.472+0.497+0.472+0.351}=\frac{10.715}{2.143}=5.000 \\
O p V a R=O p V a R_{O D}+O p V a R_{A D}=10.00
\end{gathered}
$$

Taking into account the FRMM - E1 (Weak Risk Management) and the credibility associated with the ODLV, we obtain:

$$
J_{O D, 2}=1-\frac{\frac{1}{0.5} * 0.607+\frac{3}{1} * 1.000+\frac{3}{1} * 0.607+\frac{3}{1} * 0.135+\frac{3}{1} * 0.011}{\left(\frac{1}{0.5}+\frac{3}{1}+\frac{3}{1}+\frac{3}{1}+\frac{3}{1}\right)}=1-0.462=0.537
$$




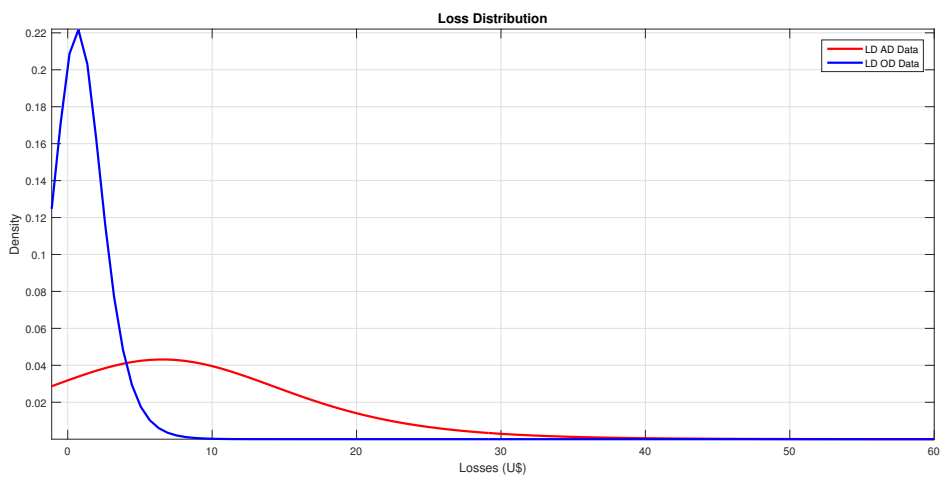

(a)

\begin{tabular}{|c|c|c|}
\cline { 2 - 3 } \multicolumn{1}{c|}{} & OD & AD \\
\hline UL & 57 & 182 \\
\hline EL & 292 & 518 \\
\hline$\sigma$ & 4.01350458 & 15.2311543 \\
\hline Media & 1.4709 & 9.5895 \\
\hline OpVar & 33.5050871 & 107.004237 \\
\hline \multicolumn{2}{|c|}{ Distribution (NLogL) } \\
\hline Loglogistic & 270.088 & 221.13 \\
\hline G.Pareto & 269.959 & 221.263 \\
\hline Lognormal & 273.145 & 221.706 \\
\hline GEV & 272.199 & 222.291 \\
\hline Weibull & 311.785 & 223.435 \\
\hline
\end{tabular}

(b)

Figure 4: Characterisation of the Loss Distribution for the OD and AD databases: (a) Loss Distributions; (b) Statistical Metrics.

For the case where the management was done with the low fuzzy set associated to ADLV, the OpVaR is calculated as:

$$
\begin{gathered}
X C_{O D, l, 1, k+1}=\frac{J_{O D, 1, k+1}}{J_{O D, 1, k}}=\frac{0.537}{0.528} * 2.5=2.542 \\
O p V a R_{O D}=\frac{0.649 * 0+0.528 * 2.542+0.503 * 5+0.528 * 7.5+0.649 * 10}{0.649+0.528+0.503+0.528+0.649}=\frac{14.307}{2.857}=5.007 \\
O p V a R_{A D}=\frac{0.351 * 0+0.472 * 2.5+0.497 * 5+0.472 * 7.5+0.351 * 10}{0.351+0.472+0.497+0.472+0.351}=\frac{10.715}{2.143}=5.000 \\
O p V a R=O p V a R_{O D}+O p V a R_{A D}=10.007
\end{gathered}
$$

Accordingly to these figures, the effect of a weak management (E1) can be observed with respect to an increase in the ODLV fuzzy sets and with regard to low losses in the ADLV. In this case, the OpVaR ranges from 10.000 to 10.007, which represents an important effect, taking in account that these figures are in thousands of dollars.

\section{Experimental Results}

Figure 4 shows the intrinsic statistical properties that characterise the AD and OD databases in the experimental design for this study. It shows that the distributions for the OD and AD have long tail properties according to the Negative Loglikelihood index. These distributions are representative for this type of risk [38], where the OpVaR for the OD is lower than for AD.

Figure 5 and 6 show the representation of the fuzzy sets for ODLV and ADLV, respectively, located in both cases at the left side of the horizontal axis. These figures show long tail distributions based on the data of the databases, a fact that is corroborated through the slope of the lines in the QQ-Plot as per Definition 1. The set of labels for each linguistic variable defined on the universe of discourse are in both cases: \{Very Low, Low, Medium, High, Very High\}.

Considering equation (25), Table 4 presents the credibility values achieved by applying the OD fuzzy sets with respect to the fuzzy set $j$ in ADLV. It can be observed that the credibility for $\mathrm{AD}$ is higher when the OD fuzzy sets have a higher degree of overlap with the fuzzy set $j$ in ADLV or when the fuzzy sets have a similar magnitude in terms of losses. 


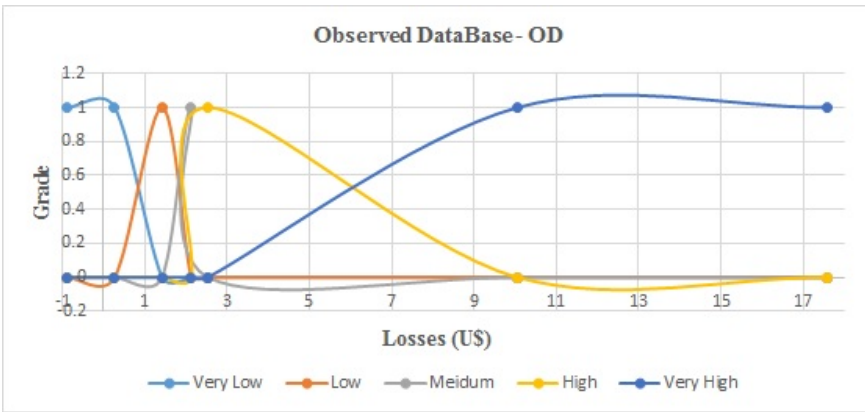

(a)

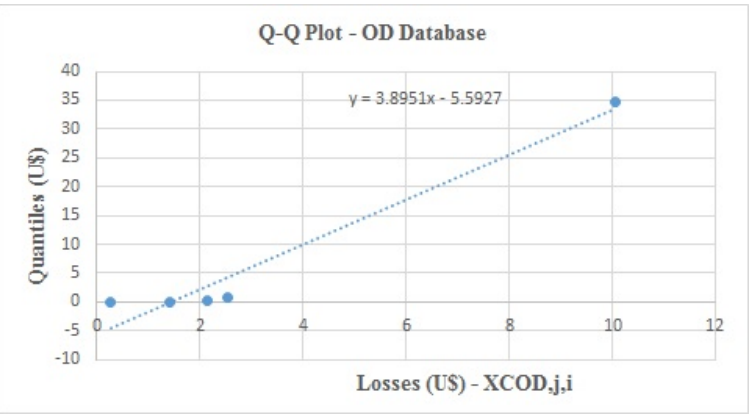

(b)

Figure 5: Loss Distribution - Observed Data: (a) ODLV; (b) QQ-Plot.

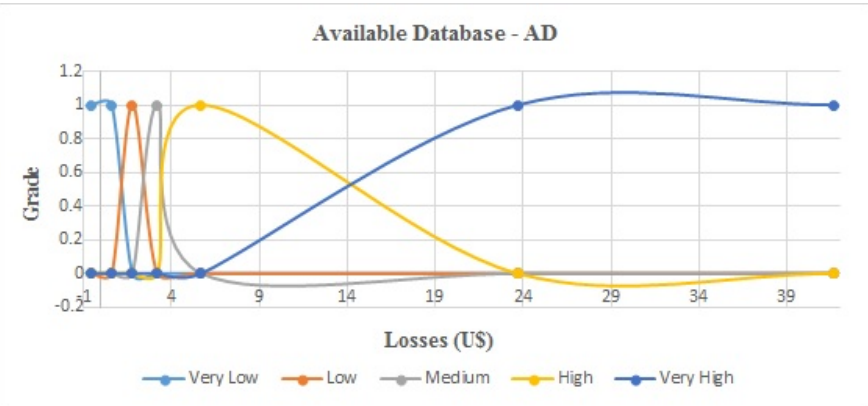

(a)

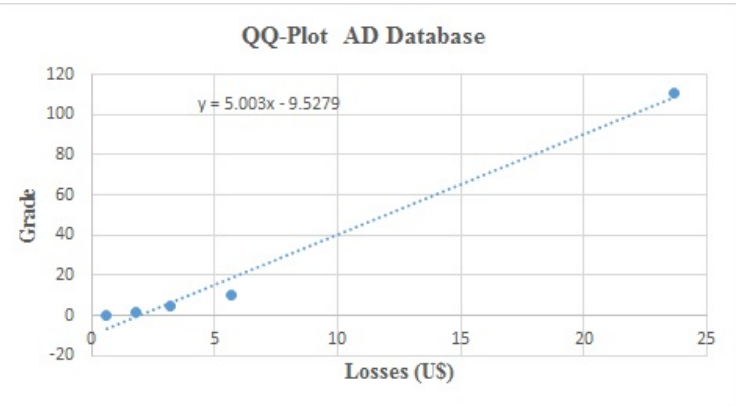

(b)

Figure 6: Loss Distribution - Available Data: (a) ADLV; (b) QQ-Plot.

\subsection{Stage 1: Credibility behaviour}

Figure 7 (a) shows that the credibility that was estimated for the OD fuzzy sets converged to unity when the OD and AD fuzzy sets have the same magnitude. Both Fig. 7 (b) and Fig. 7 (c) show that the credibility for the Very High label in ODLV decreases when the impact increases, so that the OD largest losses are automatically "rejected" by the fuzzy model when assimilating this data in the $\mathrm{AD}$ database. According to Figure 7 (c), the $V H M B$ values of the fuzzy credibility factor $\left(k_{b}\right)$ are greater than $E P V B$ values, which delivers lower values of credibility for the OD fuzzy sets with the largest losses.

Figure 8 shows the OpVaR that is estimated by applying the proposed fuzzy model and the Bühlmann model by using the process of the mean (Mean OpVaR) and the process of the variance (Variance OpVaR). The OpVaR estimated by the Bühlmann model delivered higher losses than the fuzzy model, because it uses a credibility factor to estimate the general OpVaR, when the credibility is proportional to the slope of the OpVaR line. Moreover, it can be observed that the general OpVaR estimated by the fuzzy model delivered lower losses, because this model

Table 4: Credibility factor for overlaps between $\mathrm{AD}$ and $\mathrm{OD}$ fuzzy sets, using an $I F=1$

\begin{tabular}{|l|c|c|c|c|c|}
\hline & ul,1 & ul,2 & ul,3 & ul,4 & ul,5 \\
\hline \multirow{4}{*}{} & 0.99726766 & 0.94082169 & 0.7836257 & 0.47592922 & 0.05923289 \\
\cline { 2 - 6 } & 0.9831725 & 0.99576345 & 0.89839739 & 0.58160317 & 0.06428227 \\
\cline { 2 - 6 } & 0.93953373 & 0.99623023 & 0.96044707 & 0.67685416 & 0.07345302 \\
\cline { 2 - 6 } & 0.90626478 & 0.98246134 & 0.9847665 & 0.74039998 & 0.08271061 \\
\cline { 2 - 6 } & 0.35742626 & 0.41440032 & 0.53095901 & 0.78943744 & 0.50857678 \\
\cline { 2 - 6 } & \multicolumn{5}{|c|}{ Credibility (J) } \\
\hline AD & 0.83673299 & 0.86593541 & 0.83163913 & 0.65284479 & 0.15765111 \\
\hline OD & 0.16326701 & 0.13406459 & 0.16836087 & 0.34715521 & 0.84234889 \\
\hline
\end{tabular}




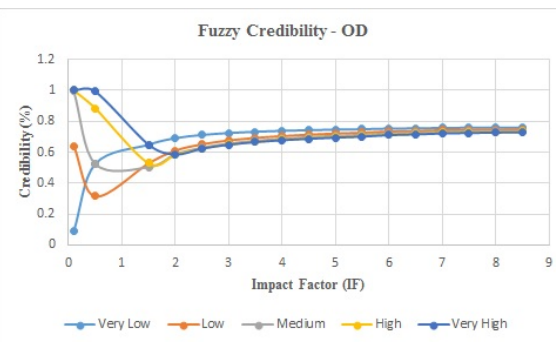

(a)

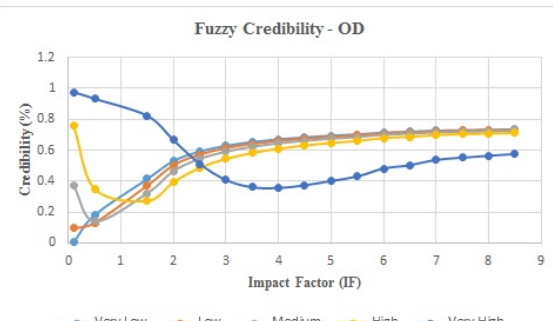

(b)

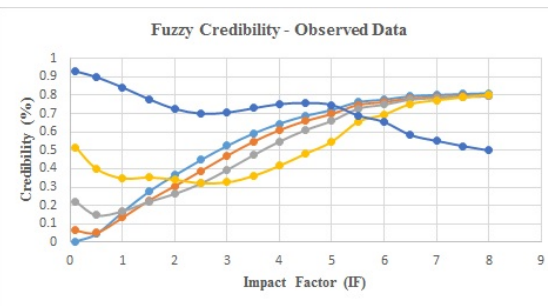

(c)

Figure 7: Estimated credibility for each fuzzy set that constitutes the observed database: (a) OD - AD Balanced fuzzy sets; (b) OD Balanced - AD Unbalanced fuzzy sets; (c) OD Unbalanced - AD Unbalanced fuzzy sets.

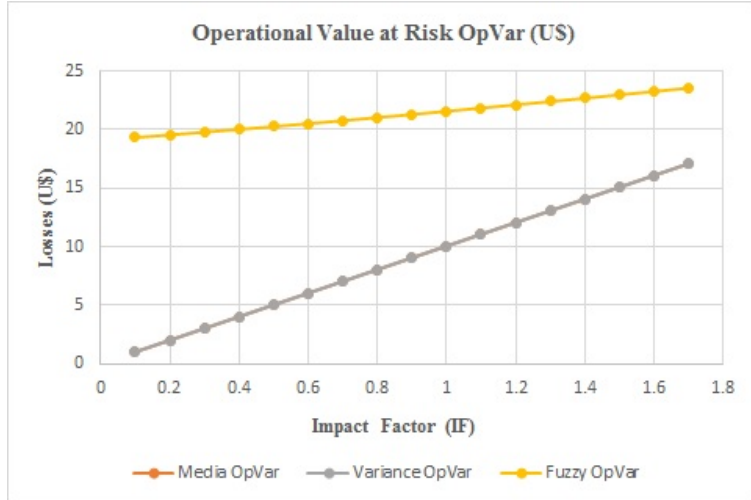

(a)

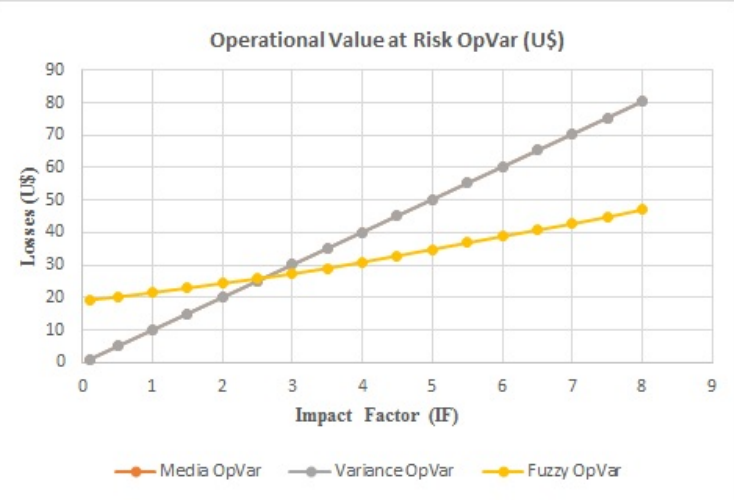

(b)

Figure 8: Operational Value at Risk: (a) Compressed Losses; (b) Expanded Losses.

uses a set of credibility factors for the corresponding OD fuzzy sets. Here, the growth of the losses was moderate, because the fuzzy model tends to estimate lower credibility indices for the largest losses in the OD database. It is important to highlight that the cut-off point between the lines shows the equilibrium between the models in estimating the OpVaR. When the OD losses are smaller, the OpVaR is estimated using the $\mathrm{AD}$ (AD credibility is higher). When the losses are bigger, the $\mathrm{OpVaR}$ is estimated using the OD database (OD credibility is bigger). This fact makes a difference when assessing analytical credibility models, where the credibility for the AD data is higher.

Figure 9 shows the evolution of LD with $I F$ values of 1 and 9 . It can be observed that the distributions evolve toward long tail distributions with heavier tails, which is corroborated by the IC-fingerprint in Table 5, where the losses are higher when the $I F$ is bigger. However, the fuzzy model keeps the mean and the OpVaR below the values of the Bühlmann analytical model. The structure and shape of the LD distributions preserve the structure and shape for different $I F$ values, which underlines the stability of the fuzzy model in estimating the OpVaR using different $I F$ values.

\subsection{Stage 2: Risk Management Matrices}

Figure 10 presents the behaviour of the proposed fuzzy model in estimating the OpVaR using different FRMMs that configure the fuzzy management model or $m$ fuzzy model. The results show that the fuzzy model with FRMMs achieved OpVaR values lower than the fuzzy model used in the previous stage, which reflects the importance of the management related to the OD data. Also, the stability of the fuzzy model in estimating the OpVaR can be observed, which is corroborated through the parallel lines. 


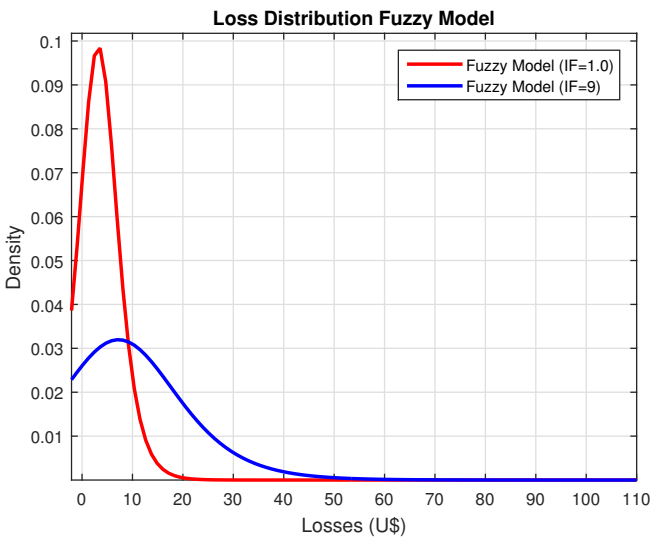

(a)

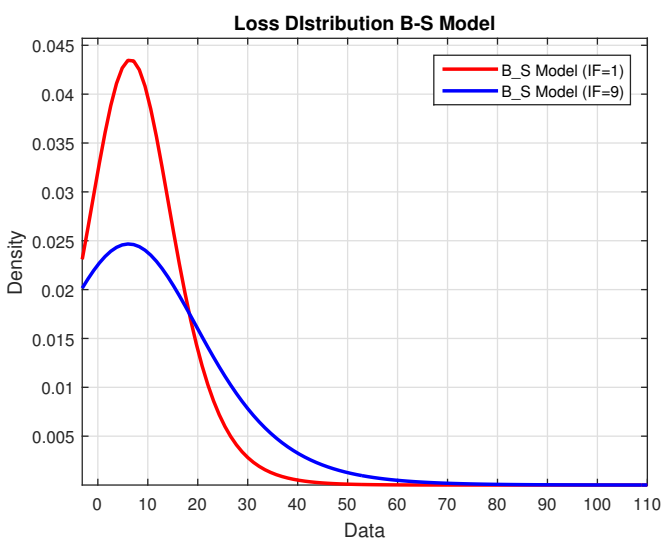

(b)

Figure 9: Evolution of the LD Distribution with $I F=1$ and $I F=9$ : (a) Fuzzy Credibility Model; (b) Bühlmann Credibility Model.

Table 5: Fingerprint LD statistical indices

\begin{tabular}{|c|c|c|c|c|c|c|}
\cline { 2 - 7 } \multicolumn{1}{c|}{} & \multicolumn{3}{c|}{ OD $I F=1$} & \multicolumn{3}{c|}{ OD $I F=9$} \\
\cline { 2 - 7 } \multicolumn{1}{c|}{} & Fuzzy & B-Variance & B-Media & Fuzzy & B-Variance & B-Media \\
\hline UL & 97 & 91 & 91 & 76 & 57 & 57 \\
\hline EL & 252 & 258 & 258 & 273 & 292 & 292 \\
\hline $\boldsymbol{\sigma}$ & 6.30921529 & 15.041344 & 15.0997045 & 26.0012683 & 36.0771405 & 36.0907955 \\
\hline Media & 4.43194404 & 9.48595864 & 9.51781526 & 12.1343187 & 13.2332572 & 13.2346888 \\
\hline OpVar & 41.4276898 & 105.641541 & 106.060659 & 218.960123 & 301.197468 & 301.304598 \\
\hline Distribution & Lognormal & Lognormal & Lognormal & Lognormal & Lognormal & Lognormal \\
\hline NLogL & 1123.775 & 1092.624 & 1093.829 & 1123.775 & 1042.263 & 1042.162 \\
\hline
\end{tabular}

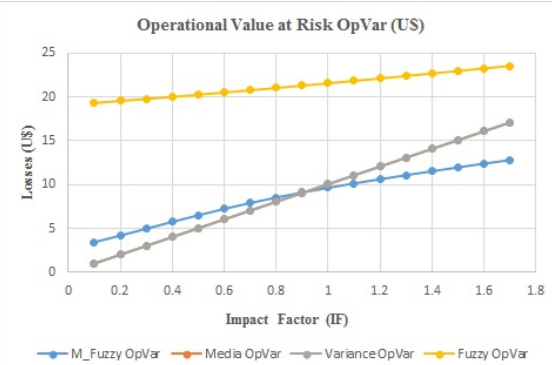

(a)

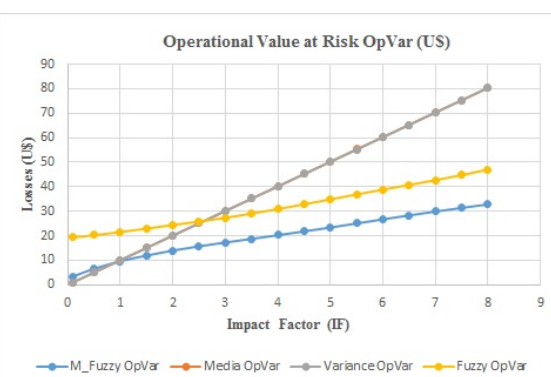

(b)

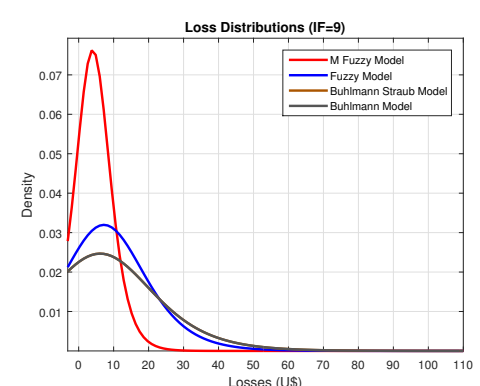

(c)

Figure 10: OpR estimated by using RCFMs: (a) Compressed losses $(I F<1)$; (b) Expanded Losses $(I F>1)$; (c) LD distributions of losses. 
Table 6: The LD distribution with $I F=9$ with FRMMS (m_fuzzy model) and without (fuzzy model and Bühlmann model).

\begin{tabular}{|c|c|c|c|c|}
\cline { 2 - 5 } \multicolumn{1}{c|}{} & \multicolumn{4}{c|}{ OD $I F=9.0$} \\
\cline { 2 - 5 } & m_Fuzzy & Fuzzy & B-Variance & B-Media \\
\hline UL & 105 & 76 & 57 & 57 \\
\hline EL & 244 & 273 & 292 & 292 \\
\hline $\boldsymbol{\sigma}$ & 8.32401839 & 26.0012683 & 36.0771405 & 36.0907955 \\
\hline Media & 5.45643251 & 12.1343187 & 13.2332572 & 13.2346888 \\
\hline OpVar & 83.1600312 & 218.960123 & 301.197468 & 301.304598 \\
\hline Distribution & Lognormal & Lognormal & Lognormal & Lognormal \\
\hline NLogL & 1180.076 & 1123.775 & 1042.263 & 1042.162 \\
\hline
\end{tabular}

Table 6 shows that the expected losses (EL) estimated using the Bühlmann mean OpVaR and the Bühlmann variance $\mathrm{OpVaR}$ for an $\mathrm{IF}=9$ were highest with heavy tails LDs, as these type of models use a general credibility factor when estimating the OpVaR, without taking into account the magnitude and impact of an risk event. Furthermore, this Figure reveals that the $m$ fuzzy model achieved the lowest OpVaR value. This is mainly due to the incorporation of FRMMs in managing the impact of the OD events. This makes the model ideal to assess the evolution of the OpVaR by using a sequence of risk profiles or FRMMs.

Figure 11 presents the evolution of the LD distribution using the sequence of risk profiles (E1 - E2 - E3). Here, the LD evolves toward more narrow distributions, with the LD distribution obtained using the E3 risk profile achieving the lowest values of risk as shown in the Figure 11 (b). Consequently, the fuzzy model can be used as a reference model in a financial organisation when evaluating a priori the effect of different risk profiles; unlike the analytical models, which do not allow to integrate risk profiles when determining operational risk.

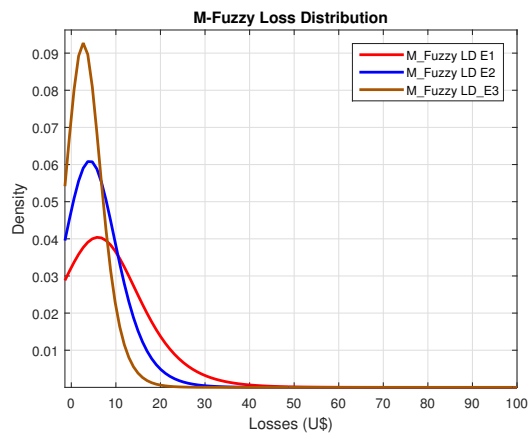

(a)

\begin{tabular}{|c|c|c|c|}
\hline & M_F LD E3 & M_F LD E2 & M_LD E1 \\
\hline UL & 81 & 94 & 90 \\
\hline EL & 268 & 255 & 259 \\
\hline $\boldsymbol{\sigma}$ & 6.79499987 & 10.7115533 & 17.9354861 \\
\hline Media & 4.12162549 & 6.32044441 & 9.46735151 \\
\hline OpVar & 42.4926595 & 77.5042355 & 142.357395 \\
\hline Distribution & Lognormal & Lognormal & Lognormal \\
\hline NLogL. & 773.023 & 922.026 & 1050.882 \\
\hline
\end{tabular}

(b)

Figure 11: Evolution of the LD distribution for different risk profiles

\section{Conclusions}

This paper presents a novel $m$ fuzzy model to assess the evolution of the OpVaR in financial organisations using a sequence of risk profiles and a set of credibility factors, defined through overlaps between fuzzy sets that represent the intrinsic properties of risk events that are stored in different databases (OD, $\mathrm{AD}$ ) and represented as linguistic variables (ODLV, ADLV). The OpVaR value obtained by the proposed model is lower with the LD characterised by a long tail, which is narrower than the corresponding estimations obtained using the analytical models 
based on Bühlmann credibility theory. This is mainly due to the control that is performed by the fuzzy risk management matrices with regard to risk events stored in the OD database.

The $m$ fuzzy model allows to assess the evolution of the LD and the OpVaR values by using a sequence of fuzzy risk management matrices (FRMMs) that show a priori the effect of a risk profile on the management of a business process in a financial organisation. This model overcomes the limitations that impose the analytical model in the integration of management matrices and using qualitative information that describes an OpR event. Different impact factors allow expanding and contracting the losses based on the registered data in the OD database.

The Expected Losses (EL) estimated by the $m$ fuzzy model were lower than the EL estimated by the analytical model. This is mainly due to the use of differentiated fuzzy credibility factors that qualitatively describe the impact of a risk event on the OpVaR, according to the label that represents a fuzzy set. While analytical models use general factors to estimate the credibility and treat risk events similarly that show different magnitudes and impacts concerning the estimation of the OpVaR.

The credibility analysis that was carried out by using unbalanced fuzzy sets shows that the credibility decreased with the expansion of the highest losses in the OD, so the estimation of the OpVaR was supported by the credibility associated with the biggest losses in the AD. This means that the organisation needs to implement risk profiles that allow to decrease the impacts of this type of risk on the OpVaR.

In terms of future work in this research area we propose the estimation of the OpVaR by applying the structure of ANFIS models, integrating into a single model the representation of the $\mathrm{OD}$ and $\mathrm{AD}$ databases as linguistic variables, the estimation of credibility by using the overlaps between fuzzy sets associated with ODLV and ADLV, respectively, and integrating different management matrices to assess a priori the impact of a risk profile on the business process of an organisation.

\section{References}

[1] Bank for International Settlements, Principles for the sound management of operational risk, http://www.bis.org/publ/bcbs195.htm (06 2011).

[2] Bank for International Settlements, Operational Risk - Supervisory Guidelines for the Advanced Measurement Approaches, http://www.bis.org/publ/bcbs196.htm (06 2011).

[3] I. Bonet, P. A. Peña, C. Lochmuller, A. Patiño, Fuzzy credibility for mixing different data sources in evaluating operational risk: Modelling operational risk, in: 2014 9th Iberian Conference on Information Systems and Technologies (CISTI), 2014, pp. 1-6.

[4] L. Zhou, D. Lu, H. Fujita, The performance of corporate financial distress prediction models with features selection guided by domain knowledge and data mining approaches, Knowledge-Based Systems 85 (Supplement C) (2015) 52 - 61.

[5] P. Dorogovs, I. Solovjova, A. Romanovs, New tendencies of management and control of operational risk in financial institutions, Procedia - Social and Behavioral Sciences 99(6) (2013) 911-918.

[6] Bank for International Settlements, Recongising the risk mitigating impact of insurance in operational risk modelling, http://www.bis.org/publ/bcbs181.htm (October 2010).

[7] Bank for International Settlements, Standardised measurement approach for operational risk - consultative document, http://www.bis.org/bcbs/publ/d355.htm (march 2016). 
[8] J. Esterhuysen, P. Styger, G. van Vuuren, Calculating operational value-at-risk (OpVaR) in a retail bank, South African Journal of Economic and Management Sciences 11 (2008) $1-16$.

[9] J. G. Catalina Bolancé, Montserrat Guillén, J. P. Nielsen, Quantitative Operational Risk Models, 1st Edition, Chapman \& Hall/CRC Finance, Chapman and Hall/CRC, 2012.

[10] E. de Jongh, R. de Jongh, D. de Jongh, G. van Vuuren, A review of operational risk in banks and its role in the financial crisis, South African Journal of Economic and Management Sciences 16 (4) (2013) 364-382.

[11] P. Sands, G. Liao, Y. Ma, Rethinking operational risk capital requirements, Working paper (2016- 6).http://www.hbs.edu/faculty/PublishingImages/behavioral-finance/ workingpapers/2016-06 $\% 20$ Rethinking $\backslash \% 200$ perational $\ \% 20$ Risk $\ \% 20$ Capital $\backslash$ $\% 20$ Requirements.pdf

[12] G. Mignola, R. Ugoccioni, E. Cope, Comments on the BCBS proposal for a New Standardized Approach for Operational Risk, ArXiv e-prints http://arxiv.org/abs/1607.00756.

[13] J. Sun, H. Li, Q.-H. Huang, K.-Y. He, Predicting financial distress and corporate failure: A review from the state-of-the-art definitions, modeling, sampling, and featuring approaches, Knowledge-Based Systems 57 (Supplement C) (2014) 41 - 56.

[14] D. D. Lambrigger. P. V. Shevchenko, M. V. Wüthrich, Data combination under Basel II and solvency 2: Operational risk goes bayesian, Bulletin of the French Actuaries 8 (16) (2008) 4-13.

[15] P. Shevchenko, Modelling Operational Risk Using Bayesian Inference, Springer Berlin Heidelberg, 2011.

[16] S. Figini, L. Gao, P. Gindici, Bayesian operational risk models, DEM Working papers series 10(2) (2015) 07-13.

[17] G. D. Aragón, F. O. Arango, F. C. Aranda, Cálculo del valor en riesgo operacional mediante redes bayesianas para una empresa financiera, Contaduría y Administración 61 (1) (2016) $176-201$.

[18] J. Duan, H. Liu, J. Zeng, Posterior probability model for stock return prediction based on analyst's recommendation behavior, Knowledge-Based Systems 50 (Supplement C) (2013) $151-158$.

[19] B. A. Ergashev, A theoretical framework for incorporating scenarios into operational risk modeling, Journal of Financial Services Research 41 (3) (2012) 145-161.

[20] J. P. Nielsen, M. Guillen, C. Bolance, J. Gustafsson, Quantitative modeling of operational risk losses when combining internal and external data, Journal of Financial Transformation 35 (2012) 179-185.

[21] G. Galloppo, D. Previati, A review of methods for combining internal and external data, Journal of Operational Risk 9 (4).

[22] R. de Jongh, T. de Wet, H. Raubenheimer, J. H. Venter, Combining scenario and historical data in the loss distribution approach: A new procedure that incorporates measures of agreement between scenarios and historical data, Journal of Operational Risk 10 (1) (2015) 45-76. 
[23] H. Bühlmann, P. V. Shevchenko, M. V. Wüthrich, A "Toy" Model for Operational Risk Quantification using Credibility Theory, The Journal of Operational Risk. 2 (1) (2009) $3-19$.

[24] R. Torresetti, G. Le Pera, A comparison of alternative mixing model for external data in operational risk, Journal of Operational Risk 10 (4) (2015) 1-21.

[25] Q. Zhang, Q. Cui, P. Chen, Multidimensional credibility estimators with random common effects and time effects, Journal of Systems Science and Complexity 30 (5) (2017) 11071120 .

[26] A. Reveiz, C. E. León, Operational risk management using a fuzzy logic inference system, Borradores de Economía 574, Banco de la República Colombia (2009).

[27] H. Izakian, A. Abraham, Fuzzy c-means and fuzzy swarm for fuzzy clustering problem, Expert Systems with Applications 38 (3) (2011) 1835 - 1838.

[28] P. Cerchiello, P. Giudici, Fuzzy methods for variable selection in operational risk management, The Journal of Operational Risk 7 (4) (2012) 25-41.

[29] V. S. Hari Rao, K. V. N. M. Ramesh, A checklist-based weighted fuzzy severity approach for calculating operational risk exposure on foreign exchange trades under the Basel II regime, Journal of Operational Risk 9 (4) (2014) 105-124.

[30] Y.-C. Ko, H. Fujita, G.-H. Tzeng, A fuzzy integral fusion approach in analyzing competitiveness patterns from wcy2010, Knowledge-Based Systems 49 (Supplement C) (2013) 1 9.

[31] F. Chiclana, F. Mata, L. G. Pérez, E. Herrera-Viedma, Type-1 OWA unbalanced fuzzy linguistic aggregation methodology: Application to eurobonds credit risk evaluation, International Journal of Intelligent Systems 33 (5) (2017) 1071-1088.

[32] F. Mata, L. G. Pérez, S.-M. Zhou, F. Chiclana, Type-1 OWA Methodology to Consensus Reaching Processes in Multi-granular Linguistic Contexts, Knowledge-Based Systems 58 (2014) 11-22.

[33] J. Wu, F. Chiclana, Visual information feedback mechanism and attitudinal prioritisation method for group decision making with triangular fuzzy complementary preference relations, Information Sciences 279 (2014) 716-734.

[34] J. Wu, F. Chiclana, A risk attitudinal ranking method for interval-valued intuitionistic fuzzy numbers based on novel score and accuracy expected functions, Applied Soft Computing 22 (2014) 272-286.

[35] L. Zhou, X. Wu, Z. Xu, and H. Fujita, Emergency decision making for natural disasters: An overview, International Journal of Disaster Risk Reduction 27 (2018) 567 - 576.

[36] Z. Hao, Z. Xu, H. Zhao, and H. Fujita, A dynamic weight determination approach based on the intuitionistic fuzzy bayesian network and its application to emergency decision making, IEEE Transactions on Fuzzy Systems PP (99) (2017) 1-1. doi: 10.1109/TFUZZ.2017.2755001

[37] Bank for International Settlements, Basel II: International Convergence of Capital Measurement and Capital Standards: a Revised Framework, https://www.bis.org/publ/ bcbs107.htm (06 2004). 
[38] A. Mora Valencia, Cuantificación del riesgo operativo en entidades financeiras en Colombia, Cuadernos de Administración 25(41) (2010) 185-211.

[39] O. Giustolisi, A. Doglioni, D. Savic, B. Webb, A multi-model approach to analysis of environmental phenomena, Environmental Modelling \& Software 22 (5) (2007) 674 - 682,

[40] M. M. Sebastian Happ, Ramona Maier, CAS: Multivariate Bühlmann-Straub credibility model applied to claims reserving for correlated run-off triangles, Variance 8 (1) (2017) $23-42$.

[41] K. Buza, A. Nanopoulos, G. Nagy, Nearest neighbor regression in the presence of bad hubs, Knowledge-Based Systems 86 (2015) 250 - 260.

[42] X. Jiang, S. Mahadevan, Y. Yuan, Fuzzy stochastic neural network model for structural system identification, Mechanical Systems and Signal Processing 82 (Supplement C) (2017) $394-411$.

[43] J. A. Hernández, J. D. Opsina, A multi dynamics algorithm for global optimization, Mathematical and Computer Modelling Volume 52 (7-8) (2010) 1271-1278.

[44] E. Mahdi, N. Ibrahim, A. Jemain, A. Kilicman, Claim dependence with common effects in credibility models with error uniform dependence, World Applied Sciences Journal 27 (12) (2013) 1551-1560.

[45] H. Q. Truong, L. T. Ngo, W. Pedrycz, Granular fuzzy possibilistic c-means clustering approach to dna microarray problem, Knowledge-Based Systems 133 (Supplement C) (2017) $53-65$.

[46] B. Di Renzo, M. Hillairet, M. Picard, A. Rifaut, C. Bernard, D. Hagen, P. Maar, D. Reinard, Operational risk management in financial institutions: Process assessment in concordance with Basel II, Software Process: Improvement and Practice 12 (4) (2007) $321-330$.

[47] T.-C. Wu, M.-F. Hsu, Credit risk assessment and decision making by a fusion approach, Knowledge-Based Systems 35 (Supplement C) (2012) 102 - 110.

[48] A. D. Ijegwa, V. O. Rebecca, F. Olusegun, O. O. Isaac, A predictive stock market technical analysis using fuzzy logic., Computer and Information Science 7 (3) (2014) 1-17.

[49] F. De Martino, F. Gentile, F. Esposito, M. Balsi, F. Di Salle, R. Goebel, E. Formisano, Classification of fmri independent components using ic-fingerprints and support vector machine classifiers, NeuroImage 34 (1) (2007) 177-194.

[50] B. Cooper, B. Piwcewicz, N. Warren, Operational risk modelling: how fare we progressed, in: Financial Services Forum, Actuaries Institute, 2014, pp. 2-27.

[51] D. R. Cox, Partial likelihood, Biometrika 62 (2) (1975) 269-276. 